\title{
Cholesterol Deposit
}

National Cancer Institute

\section{Source}

National Cancer Institute. Cholesterol Deposit. NCI Thesaurus. Code C54097.

Deposition of cholesterol in the skin. It appears as a slightly elevated and yellow bump usually in the skin around the eyes. 